\title{
The effect of two exercise protocols on lumbar spine sagittal range of motion
}

Article in Journal of Back and Musculoskeletal Rehabilitation · March 2014

Impact Factor: $0.71 \cdot$ DOI: 10.3233/BMR-140465 · Source: PubMed

READS

34

7 authors, including:

\section{Emma Sly}

The Royal Marsden NHS Foundation Trust

2 PUBLICATIONS 0 CITATIONS

SEE PROFILE
Fiona Wilson

Trinity College Dublin

63 PUBLICATIONS 175 CITATIONS

SEE PROFILE 


\title{
The effect of two exercise protocols on lumbar spine sagittal range of motion
}

\author{
Emma Sly $^{\mathrm{a}, *}$, Cliona Coleman ${ }^{\mathrm{b}}$, Daniel Quinn ${ }^{\mathrm{b}}$, Stephen Mark Armstrong ${ }^{\mathrm{b}}$, Chris Garvey $^{\mathrm{b}}$, \\ Ciara O'Donoghue ${ }^{\mathrm{b}}$ and Fiona Wilson ${ }^{\mathrm{b}}$ \\ ${ }^{\mathrm{a}}$ Herts and Essex Community Hospital, Bishops Stordford, Herts, UK \\ ${ }^{\mathrm{b}}$ School of Physiotherapy, Trinity College Dublin, Trinity Centre for Health Sciences, Dublin, Ireland
}

\begin{abstract}
.
BACKGROUND: Current research has shown that exercise induces an increase in spinal range of motion (ROM) which is primarily due to spinal creep. However, there is a lack of consensus regarding the cause of spinal creep; some believe it is due to the warm up effect of exercise while others believe it is the result of the position of the lumbar spine during the exercise.

AIM: The aim of this prospective study is to investigate first whether a change in lumbar spine ROM is seen following exercise and second whether a greater change in ROM is seen following a fatiguing protocol in a seated position or in an upright position. METHODS: Fifteen healthy individuals aged between 18 and 35 years volunteered to participate in the study. Range of motion was assessed with an electro-goniometer prior to and following two exercise tests which lasted for a period of nine minutes each. Submaximal protocols for the treadmill and bicycle were used.

RESULTS: No significant change in lumbar spine ROM was detected following the bicycle test $(p=0.301)$ or the treadmill test $(p=0.132)$ implying that the warm up effect of exercise has little impact on lumbar spine ROM. Likewise, no significant difference was seen in the changes following exercise on the bicycle and the treadmill, implying that position also has little effect on ROM.

CONCLUSION: The findings of this study contradict those of previous research where an increase in lumbar spine ROM was seen following exercise. Further research using a large scale, heterogeneous cohort is needed to further determine the effects of exercise on lumbar spine ROM.
\end{abstract}

Keywords: Lumbar spine, range of motion, creep, position, exercise

\section{Introduction}

Low back pain (LBP) is the most common cause of long term disability in Western industrialised countries with a lifetime prevalence of $90 \%[1,2]$. It is well documented that repetitive loading of the lumbar spine contributes to the development of low back pain [3]. Loading of the lumbar spine through a combination of compression, bending and torsion can occur with many activities of daily living (ADL). Specific work postures can place individuals at a higher risk of developing

*Corresponding author: Emma Sly, Herts and Essex Community Hospital, Bishops Stordford, Herts, United Kingdom, CM23 5JH. Tel: +44 7864917622;:E-mail:slye@tcdie
LBP. An epidemiological study found a positive correlation between agricultural and construction workers, who regularly adopt a flexed posture, and the presence of LBP [4].

Likewise many sporting activities require repetitive movements of flexion, hyperextension and torsion such as rowing, gymnastics and cycling. With sustained loading of the spine in these positions creep can occur. Wilson et al. found that lumbar spine flexion ROM increased significantly $\left(4.4^{\circ} \pm 0.9^{\circ}\right)$ in 19 male rowers following an ergometer trial where participants were subject to repeated flexion [5]. Similarly, Sanchez et al. reported a significant increase in lumbar spine flexion $\left(2.3^{\circ} \pm 2.5^{\circ}\right)$ in 15 healthy volunteers who maintained a flexed sitting position for one hour [6]. 


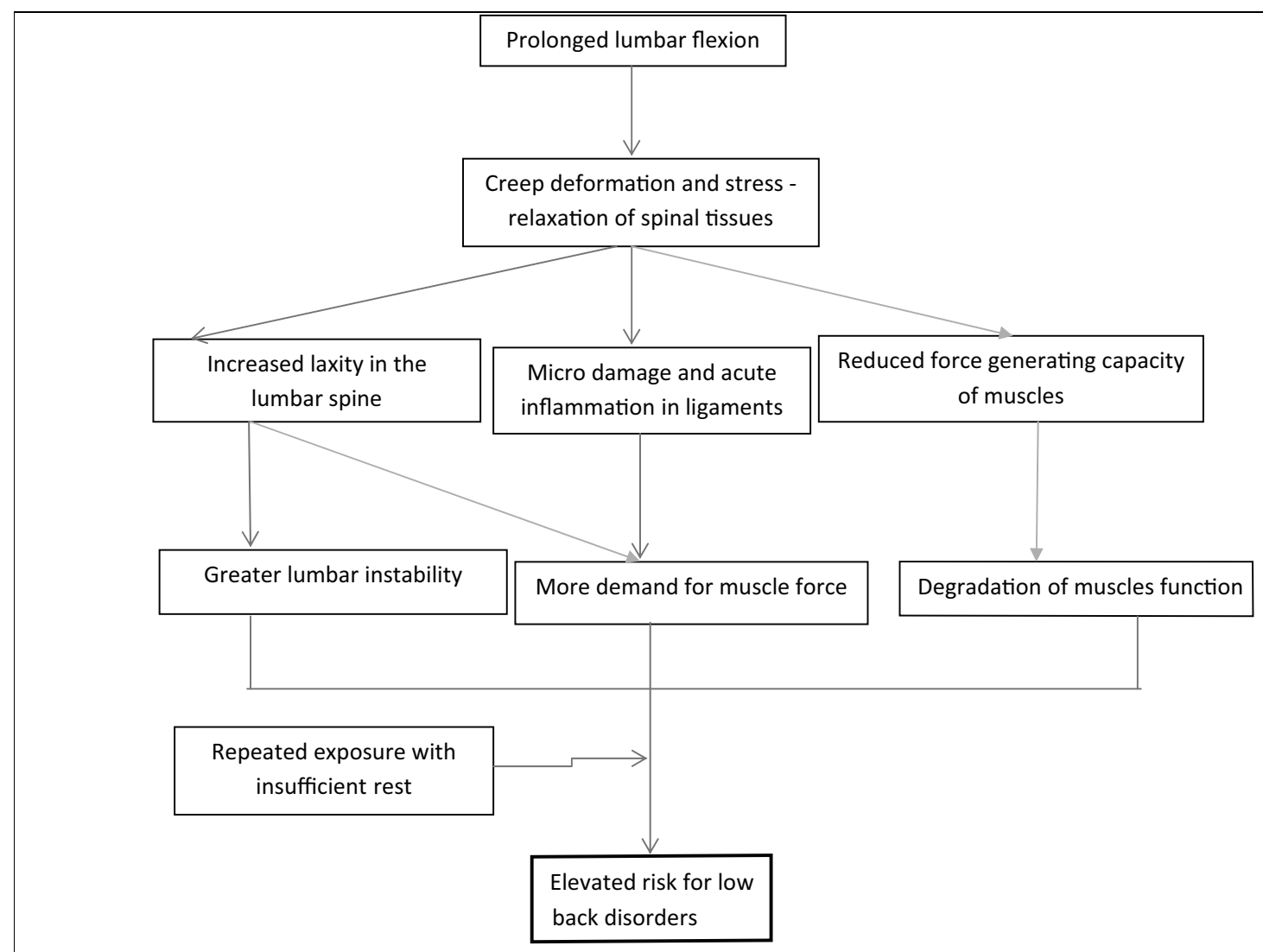

Fig. 1. Proposed effects of spinal creep.

Adams and Dolan noted that if a flexed posture is maintained the passive tissues (inter vertebral disks (IVD), ligaments and capsules) will deform at a slow rate due to their viscoelastic properties. More specifically, the authors noted that, "creep arises from a time dependent expulsion of water from spinal tissues, especially the intervertebral disks resulting in a loss of disk height and increased slack in the posterior ligaments' [7]. Thus creep causes increased laxity in the spinal structures with a resultant change in lumbar spine ROM. Moreover, the creep deformation places extra stress on the active tissues (spinal and abdominal muscles), in an attempt to increase the overall stability of the spine [8]. When the spine is subject to loading and creep occurs, it takes a significantly longer time for the viscoelastic tissues to recover from this loading. It was noted that in feline specimens, seven hours of rest was required for the viscoelastic tissues to fully recover following 20 minutes of cyclic or static loaded flexion [9]. Furthermore McGill and Brown noted that 20 minutes of a sustained flexed posture, followed by 20 minutes of rest resulted in the musculature recovering only $50 \%$ of their pre creep magnitude [10]. In this vulnerable recovery period the spine is more prone to injury. The proposed effects of creep are summarised in Fig. 1.

While some authors suggest that creep is due to the position of the spine during the activity, others propose it is a result of the 'warm up' effect of exercise. Ensink et al. stated that: "The warming up of muscles results in a change in the structure of the disc; diffusion of fluid out of the nucleus pulposus, leading to a decrease in tension of the anterior and posterior ligaments and muscles, resulting in an increased lumbar ROM" [11].

Knowledge regarding the cause of creep would be of great value considering aerobic exercise has been shown to be an important element in the treatment of LBP [2]. Exercising in a way that induces the least amount of spinal creep would be preferable to limit the risk of further damage to the spine.

There is a considerable amount of research in the area of spinal creep and loading of the lumbar spine in various positions. However, few studies have compared the warm up effect of exercise and position on lumbar spine ROM. This study aims to narrow this gap by examining the effect of two fatiguing protocols on 
the lumbar spine ROM. It is recognised that creep is a complex and anatomical phenomenom and thus can be difficult to measure; although the most simple objective measure is lumbar ROM. Thus, for the purposes of this study, lumbar ROM will be used as a proxy measure for creep. The objectives of the study are:

- To investigate whether exercise results in an increase in lumbar spine ROM in moderately active individuals.

- To investigate whether exercise on a bicycle in a seated position (flexed lumbar spine) induces greater change in ROM than walking (neutral lumbar spine).

It was hypothesised that lumbar spine ROM would increase following both fatiguing protocols and that there would be a greater increase in ROM following exercise in a seated position due to the effect of sustained flexion on the lumbar spine.

\section{Methodology}

\subsection{Participants}

Subject Recruitment: Healthy, physiotherapy students aged between 18 and 35 were recruited by email advertisement through the Discipline of Physiotherapy, Trinity College, Dublin. Volunteers who responded were given a participant information leaflet outlining the details of the study. A period of seven days was permitted for volunteers to reflect on the information and ask questions prior to participation.

\subsection{Inclusion criteria}

Participants were included in the study if they were:

- Over 18 years

- Moderately active according to the Baecke Physical Activity Questionnaire [12,13].

\subsection{Exclusion criteria}

Participants were unable to partake in the study if one or more of the following were present:

- Present musculoskeletal injury

- Illness or infection at the time of testing

- History of lumbar spine injury in the previous 6 months

\begin{tabular}{lcc}
\multicolumn{3}{c}{ Table } \\
& Participant characteristics \\
\hline Variable & Mean \pm SD & Range \\
\hline Age (years) & $23.75 \pm 3.54$ & $21-33$ years \\
Height (centimetres) & $176.59 \pm 8.96$ & $163-190 \mathrm{~cm}$ \\
Weight $(\mathrm{kg})$ & $74.22 \pm 7.25$ & $64.8-84.25 \mathrm{~kg}$ \\
BMI $\left(\mathrm{kg} / \mathrm{m}^{2}\right)$ & $23.78 \pm 1.88$ & $19.9-27.2$ \\
BPAQ Score & $7.51 \pm 1.23$ & $5.25-9.67$ \\
\hline
\end{tabular}

Age and anthropometric data for all subjects $(n=15) . \mathrm{SD}=\mathrm{Stan}-$ dard Deviation, $\mathrm{kg}=$ kilogrammes, $\mathrm{kg} / \mathrm{m}^{2}=$ kilogrammes per meter squared, $\mathrm{BMI}=$ body mass index, $\mathrm{BPAQ}=$ Baecke Physical Activity Questionnaire.

A total of 16 subjects volunteered to participate in the study. Of the initial 16 participants recruited only 15 (6 female, 9 male) completed the study. One participant withdrew due to an acquired musculoskeletal injury. Descriptive data for the remaining 15 participants are summarised in Table 1. All subjects were deemed to be at least moderately active according to the BPAQ Score, ranging from 5.25-9.67.

\subsection{Ethical approval}

Ethical approval was attained from the Faculty of Health Science Research Ethics Committee, Trinity College Dublin. Written informed consent was obtained from all participants prior to study commencement. Participants were provided with no compensation. Confidentiality was ensured by assigning each participant an individual number, by which they were identified throughout the study period. Data were stored in secure computer file protected by a password known only to the lead investigator. Participant records were stored in a secure location, which only the research team had access to.

\subsection{Equipment and preparation}

Prior to testing height and weight was assessed with the Seca Stadiometer and Beam Scale (Vogel and Heike, Model Number 7101021009). Body mass index (BMI) was calculated with this information. All testing was performed in a practical room in the Trinity College Centre for Health Sciences.

The Baecke Physical Activity Questionnaire was used to assess baseline levels of physical activity. All participants were required to be moderately active to participate in the study as indicated by a score ranging between 4.2-10.6. This questionnaire has been shown to have good validity and reliability $[12,13]$.

Electro-goniometer: Sagittal motion of the lumbar spine was measured using a flexible, twin axis, 
4 E. Sly et al. / The effect of two exercise protocols on lumbar spine sagittal range of motion

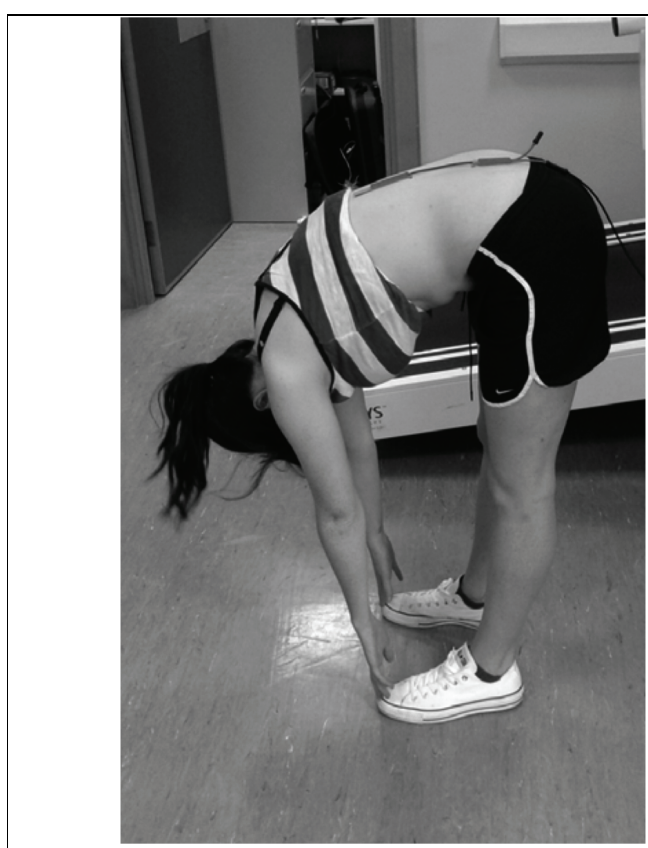

Fig. 2. Assessing ROM with the electrogoniometer.

SG150B electrogoniometer (Biometrics Ltd, Gwent, UK) connected to a data logger system (Biometrics DataLog P3X8). This has proven to be a valid and reliable measuring tool [14]. One individual from the research team was responsible for assessing ROM and was deemed competent in this task by the supervising academic member of staff. Before use, the instrument was verified against a Universal Goniometer, showing excellent agreement at the angles recorded in this study with a maximum inherent error of $0.4^{\circ}$.

\subsection{Procedure}

The electrogoniometer when attached to the skin only covered two joints (three vertebrae), thus the midlumbar spine was chosen as this is the area where the greatest degree of sagittal flexion is observed [14,15]. The upper electrogoniometer end block was placed over the spinous process of L 2 and the lower end block was placed over the spinous process of L4. Prior to applying the electrogoniometer, the skin was sprayed with 'Tuf-Skin' (Cramer Products Inc., Kansas, USA) to reduce slippage of the blocks. The blocks were placed while the participant was standing, and were secured with double sided tape. The end blocks were then secured with tape and an outline of their position was marked on the skin. The electro goniometer was fitted in standing with the feet shoulder width apart.

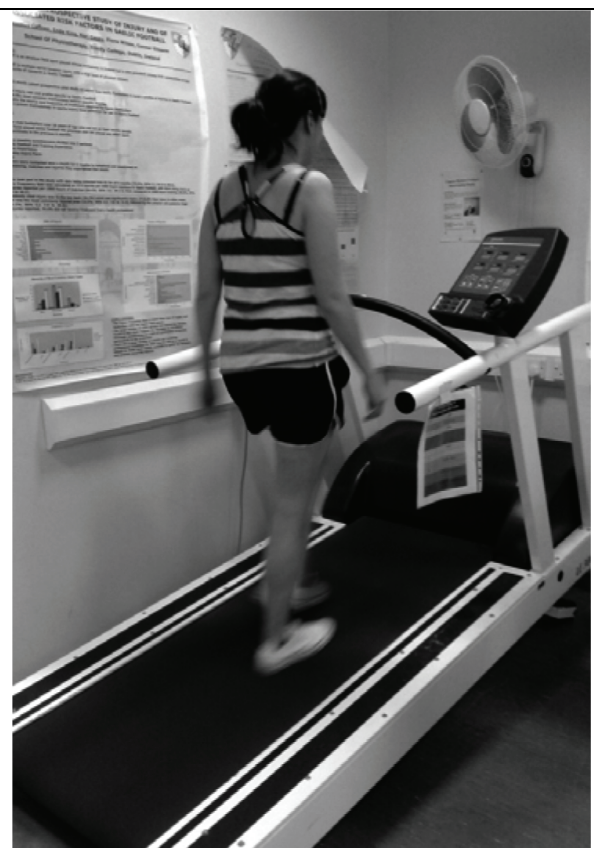

Fig. 3. Treadmill set Up.

Range of motion was assessed pre and post exercise testing. Standardised verbal cues were given to each participant, 'Bend down to touch your toes and keep your knees straight.' This position was held for three seconds while a reading was taken from the electro goniometer. The procedure was repeated three times, each range was recorded and the mean was used for statistical analysis (Fig. 2). The end blocks were repositioned into place if they had moved during the exercise. The electro-goniometer was zeroed between each participant.

\subsection{Intervention}

The intervention was designed so that each participant performed two sub-maximal exercise tests; one in a seated position (stationary bicycle ergometer) and one on a treadmill (Fig. 3). The exercise tests were performed a week apart. Subjects were asked to abstain from alcohol or strenuous activity 24 hours prior to testing.

\subsubsection{PWC 170 submaximal test}

Participants were fitted with a heart rate monitor (Polar Electro, Finland) and were given instructions regarding the test procedure. Participants then performed a sub maximal exercise test on a stationary bicycle (Monark Ergomedic 874E) according to a modified 
E. Sly et al. / The effect of two exercise protocols on lumbar spine sagittal range of motion

\begin{tabular}{|c|c|c|c|c|}
\hline \multicolumn{5}{|c|}{$\begin{array}{c}\text { Table } 2 \\
\text { Modified PWC170 Protocol }\end{array}$} \\
\hline Stage & Load & $\begin{array}{l}\text { Duration } \\
\text { (mins) }\end{array}$ & $\begin{array}{l}\text { Predicted HR } \\
\text { (end of } 3 \mathrm{~min} \text { ) }\end{array}$ & $\begin{array}{c}\text { Pedal } \\
\text { revolution speed }\end{array}$ \\
\hline 1 & $60 \mathrm{w}$ & 3 mins & $120 \mathrm{bpm}$ & $60 \mathrm{rpm}$ \\
\hline 2 & $72 \mathrm{w}$ & 3 mins & $150 \mathrm{bpm}$ & $60 \mathrm{rpm}$ \\
\hline 3 & $96 \mathrm{w}$ & 3 mins & $170 \mathrm{bpm}$ & $60 \mathrm{rpm}$ \\
\hline
\end{tabular}

Initial workload is set at one watt per $\mathrm{kg}$ of body weight. Further increases are made according to heart rate. An example of a $60 \mathrm{~kg}$ individual given above. Data for calculating initial loading and subsequent increases provided by Eurofit, (1993), Eurofit Tests of Physical Fitness, 2nd Edition, Strasbourg pp. 30-40.

Table 3

Modified bruce protocol

\begin{tabular}{cccc}
\hline Stage & Speed $(\mathrm{km} / \mathrm{hr})$ & Grade $(\%)$ & Duration $(\mathrm{min})$ \\
\hline 1 & 4.5 & 10 & 3 \\
2 & 4.5 & 12 & 3 \\
3 & 4.5 & 14 & 3 \\
\hline
\end{tabular}

$\mathrm{km} / \mathrm{hr}=$ kilometres per hour, grade $(\%)=$ treadmill incline.

PWC170 protocol (Table 2). The bicycle seat height was adjusted so that each participant's knee joint was at $15^{\circ}$ when the pedal was at the lower position. Throughout the test participants maintained a flexed lumbar spine by keeping their upper limbs supported on the handle bars. Speed remained constant throughout the nine minutes, while the resistance increased incrementally according to heart rate response. The PWC 170 protocol has shown to be a valid and reliable exercise measure for aerobic power [17].

\subsubsection{Modified bruce protocol}

Participants were given instructions regarding the treadmill test procedure. A modified version of the Bruce protocol was employed to replicate the PWC 170 protocol on the stationary bicycle [18]. The test began at a speed of $4.5 \mathrm{~km} / \mathrm{hr}$ and at an incline of $10^{\circ}$. Speed remained constant throughout the test while the incline increased every three minutes. The protocol is outlined in Table 3.

\section{Data analysis}

All the data was entered into and analysed using Microsoft ${ }^{\mathrm{TM}}$ Excel 2010. Anthropometric measurements were inputted and the mean and standard deviation were calculated for patient characteristics. A paired t-test was used to determine whether there was a difference between lumbar spine ROM prior to and following each exercise protocol. A value of $\mathrm{P} \leqslant 0.05$ was used as an indicator of statistical significance. To make a comparison of ROM changes (pre versus post)
Mean ROM (degrees) pre and post test; PWC-170 compared with Bruce protocol

\begin{tabular}{cccccc}
\hline & \multicolumn{2}{c}{ PWC 170 } & & \multicolumn{2}{c}{ Bruce } \\
\cline { 2 - 3 } \cline { 5 - 6 } Participant & Pre test & Post test & & Pre test & Post test \\
\hline 1 & 37.3 & 39 & & 40.3 & 40.6 \\
2 & 49.6 & 51 & & 50.3 & 47 \\
3 & 46.3 & 49.6 & & 46.6 & 47.6 \\
4 & 40.3 & 63.3 & & 53.3 & 66.6 \\
5 & 48 & 52 & & 46.6 & 53.6 \\
6 & 43.3 & 46.3 & & 31.3 & 40 \\
7 & 41.3 & 54.6 & & 47.6 & 42.3 \\
8 & 51 & 45.3 & & 46 & 43 \\
9 & 54.3 & 45.6 & & 55 & 72.3 \\
10 & 48.7 & 43.3 & & 36 & 45 \\
11 & 45 & 44.3 & & 41 & 54 \\
12 & 42.3 & 44.3 & & 53 & 50 \\
13 & 53.6 & 55 & & 61.3 & 62.3 \\
14 & 51.3 & 53 & & 50 & 42.3 \\
15 & 75 & 72.7 & & 65 & 64 \\
Mean & 48.5 & 50.6 & & 48.2 & 51.4 \\
SD & 8.9 & 8.6 & & 8.9 & 10.4 \\
t-test & & $P=0.3$ & & $P=0.1$ \\
\hline
\end{tabular}

between protocols, an ANOVA would be the test of ${ }_{228}$ choice, however as it was not possible to fully stan- 229 dardise end block placement between testing days, it 230 was decided not to compare absolute values but rather $\quad 231$ change scores (pre versus post ROM). Thus a paired 232 t-test was used to compare the change following the 233 PWC-170 with the change following the Bruce proto- $\quad 234$ col.

\section{Results}

The mean values for ROM prior to and following both fatiguing protocols are given in Table 4 . A paired student t-test showed that the change in ROM (pre-test compared with post-test) following the PWC 170 protocol was a small mean increase of $2.1^{\circ}$ which was not significant ( $p=0.3$ ). Similarly, a small change in ROM was seen following the Modified Bruce protocol of $3.2^{\circ}$ but this was not statistically significant $(p=$ $0.1)$.

When the mean change in ROM following the PWC170 was compared with the Bruce protocol (summarised in Table 5) there was no significant differences in lumbar ROM changes between the testing protocols $(p=0.7)$.

Participants had varying responses to the exercise protocols; seven out of fifteen participants showed an increase in ROM following one exercise protocol but a decreased following the other. Only six participants showed an increase in ROM following both exercise 
6 E. Sly et al. / The effect of two exercise protocols on lumbar spine sagittal range of motion

\begin{tabular}{|ccc}
\hline \multicolumn{3}{|c}{ Table 5 } \\
$\begin{array}{c}\text { Mean changes in ROM (degrees) following exercise; } \\
\text { pared with Bruce protocol }\end{array}$ \\
\hline Participant & Change PWC170 com- & Change bruce \\
\hline 1 & 1.7 & 0.3 \\
2 & 1.4 & -3.3 \\
3 & 3.3 & 1.0 \\
4 & 23.0 & 13.3 \\
5 & 4.0 & 7.0 \\
6 & 3.0 & 8.7 \\
7 & 13.3 & -5.3 \\
8 & -5.7 & -3.0 \\
9 & -8.7 & 17.3 \\
10 & -5.4 & 9.0 \\
11 & -0.7 & 13.0 \\
12 & 2.0 & -3.0 \\
13 & 1.4 & 1.0 \\
14 & 1.7 & -7.7 \\
15 & -2.3 & -1.0 \\
Mean & 2.1 & 3.2 \\
SD & 7.7 & 7.7 \\
t-test & $P=\mathbf{0 . 7}$ & \\
\hline
\end{tabular}

tests (Mean increase $5.6^{\circ} \pm 6.4^{\circ}$ ). Two participants showed a decrease in ROM following both exercise tests (Mean decrease $3^{\circ} \pm 1.9^{\circ}$ ).

\section{Discussion}

The aim of the study was to investigate the mode of exercise which minimises or exacerbates creep in the lumbar spine. Specifically, this involved investigating whether a change in lumbar spine ROM is seen following exercise and whether a greater change is seen following a fatiguing protocol in a seated position or during an upright position. Exercising in a way that induces the least amount of spinal creep would be preferable to limit the risk of further damage to an already vulnerable spine.

The results from this study indicate that exercise had only a small effect on lumbar spine ROM. Following a nine minute, incremental, sub maximal exercise test on a stationary bicycle a non-significant increase in range of motion was detected ( $p=0.3$ ). This contradicts previous research which suggests that when a flexed posture is maintained creep occurs. This may in part be explained by constant activation of the spinal muscles while cycling. Burnett et al. (2004) used EMG activity to show that there was constant activation of bilateral rectus abdominus, external oblique, internal oblique, lumbar multifidus and erector spinae while cycling on a stationary bicycle [19]. Wilke et al. (1999) stated that muscle activity results in fluctuating pressures on the intervertebral disks, promoting the flow of fluid, and ultimately reducing the likelihood of creep [21]. Moreover, in the seated position with the upper limbs supported on the handle bars, the pressure placed on the intervertebral disks is reduced as the upper limbs support a portion of the body weight. Intradiscal pressure was found to be $0.43 \mathrm{Mpa}$ during sitting with the elbows supported on the thighs, while bending forward while sitting without upper limb support increased intradiscal pressure to $0.83 \mathrm{Mpa}$ [21].

Likewise, a non-significant change in ROM was seen following the submaximal treadmill test $(p=$ 0.1 ). These results contradict White et al. who found that running for a distance of nine miles significantly decreased vertebral column height and increased lumbar spine ROM [22]. The authors stated that during running there is significant compressive pressure placed on the spine as force is transmitted through the lower extremities, pelvis and to the vertebra. The repetitive and compressive axial load placed on the disks can cause water to extrude from the nucleus pulposus resulting in a loss in disk height. The loss in disk height increases intervertebral joint laxity resulting in an increase in ROM. These effects are not directly comparable with the present study as participants exercised for a different length of time ( 9 minutes versus $59 \mathrm{~min}$ utes).

More recently, the effect of cycling was compared with walking regarding spinal shrinkage. Results showed greater creep during walking than cycling, ( $-7.9 \mathrm{~mm}$ and $-3.7 \mathrm{~mm}$ respectively) [23]. While the change in ROM in the present study did not meet statistical significance there was a trend towards a larger increase in ROM following the treadmill test which is in agreement with the findings of White et al. [22] and van Deursen et al. [23].

There are several limitations to this study that could have potentially affected the results. The interpretation of the results is limited to undergraduate physiotherapy students who were deemed physically fit according to the Baecke Physical Activity Questionnaire and thus do not conform to the demographics of a usual population. The majority $(n=14)$ of participants were within the age bracket of 20-30 years which has been shown to have an effect on creep rate. It was noted that the young and those with mild degenerative changes in the intervertebral disks will lose less height during exercise due to the higher water content of the nucleus populous and the better compression ability of the disks [25]. Busscher et al. concurred with this, stating that a larger amount of creep occurs in older people when placed under the same stresses [26]. Further- 
more, the diurnal variations known to affect creep were not accounted for [28,29]. All testing was done in the morning but the time of rising was not recorded for each participant prior to each exercise test.

In addition to the physiological factors, there were mechanical factors that may have influenced the results as well. The treadmill protocol required the incline be increased incrementally $\left(10^{\circ}, 12^{\circ}, 14^{\circ}\right)$ to increase the workload. However, walking at an incline increases lumbar flexion in the spine as the pelvis is moved into a posteriorly tilted position. The degree of flexion obtained while sitting on the stationary bicycle may not have been that different from the flexion caused by walking up the set incline. Considering this, it is not surprising that there was no significant difference between the change in ROM seen in the two protocols $(p=0.7)$. However, these factors must be considered when prescribing exercise to individuals with a history of disk bulge as seated exercise and mobilising on an incline results in flexion of the lumbar spine and increased pressure on the nucleus pulposus. As previously noted, Solomonow et al. stated that twenty minutes of sustained or cyclic loading has been shown to induce creep in the lumbar spine. As creep is a time dependant phenomenon, the 'dosage' delivered in this study may simply have been insufficient to cause a change in ROM [9].

A further limitation to acknowledge is the limited accuracy of the instrument, particularly when compared to traditional motion analysis equipment. However, the relevance of this study to the journal readership (many of whom are clinicians) is that a portable tool has been used which is relatively inexpensive compared to traditional motion analysis equipment; this tool could be used in clinical practice in conditions beyond a lab such as the community. This study has demonstrated that the change in spinal position over time can be measured in such a way which may be of interest to those prescribing aerobic exercise to low back pain patients or giving ergonomic advice as part of exercise prescription.

\section{Conclusion}

Previous research has shown that walking places stress on the nucleus pulposus resulting in diffusion of fluid out of the disc leading to decrease in tension of the anterior and posterior ligaments and muscles, resulting in increased lumbar ROM. Cycling with the upper limbs supported appeared to place less stress on the intervertebral disks, resulting in less creep. However, in the present study neither the bicycle nor the treadmill test resulted in a statistically significant change in lumbar ROM indicating that moderate exercise for a period of nine minutes does not result in creep and does not place individuals at further risk of back injury. Due to the limitations discussed earlier, further research is required to fully understand the effects of exercise on spinal biomechanics.

\section{References}

[1] Andersson GB. Epidemiological features of chronic low back pain. Lancet. 1999; 354: 581-585.

[2] Henchoz Y, Goumoe P, Norberg M, Paillex R, So AK. Role of physical exercise in low back pain rehabilitation. A randomized controlled trial of a three-month exercise program in $\quad 398$ \begin{tabular}{l|r}
\hline patients who have completed multidisciplinary rehabilitation. & 399 \\
\hline
\end{tabular} \begin{tabular}{l|l} 
Spine . 2010; 35(12): 1192-1199. & 400
\end{tabular}

[3] Shin G, D'Souza C, Lui YH. Creep and fatigue development 401 in the low back in static flexion. Spine. 2009; 34(17): 1873- 402 1878.

[4] Rosecrance J, Rodgers G, Merlino L. Low back pain and 404 musculoskeletal symptoms among Kansas farmers. American 405 \begin{tabular}{|l|l}
\hline Journal of Industrial Medicine. 2006; 49: 547-56. & 406
\end{tabular}

[5] Wilson F, Gissane C, Gormley J, Simms C. Sagittal plane 407 \begin{tabular}{lll}
\hline motion of the lumbar spine during ergometer and single & 408 \\
\hline
\end{tabular} \begin{tabular}{lll}
\hline scull rowing. Sports Biomechanics. 2012; DOI: 10.1080/ 409 \\
\hline
\end{tabular} \begin{tabular}{|l|l}
\hline 14763141.2012 .726640$. & 410 \\
\hline
\end{tabular}

[6] Sanchez-Zuriaga D, Adams MA, Dolan P. Is activation of the $\overline{411}$ \begin{tabular}{|l|l}
\hline back muscles impaired by creep or muscle fatigue? Spine. & 412 \\
\hline
\end{tabular} 2010; 35(5): 517-527.

[7] Adams MA, Dolan P. Time-dependent changes in the lumbar 4 \begin{tabular}{l|r}
\hline spine's resistance to bending. Clinical Biomechanics. 1996; & 415 \\
\hline
\end{tabular} \begin{tabular}{l|l} 
11: 194-200. & 416 \\
\hline
\end{tabular}

[8] $\overline{\text { McGill SM, Grenier S, Kavcic N, Cholewicki J. Coordina- }} \overline{417}$ \begin{tabular}{ll}
\hline tion of muscle activity to assure stability of the lumbar spine. & 418 \\
\hline
\end{tabular} \begin{tabular}{l|r}
\hline Journal of Electromyography and Kinesiology. 2003; 13(4): & 419 \\
\hline $353-359$.
\end{tabular} 353-359.

[9] Solomonow M, Baratta RV, Zhou BH, Burger E, Zieske A, 421 Gedalia A. Muscular dysfunction elicited by creep of lumbar 422 viscoelastic tissue. Journal of Electromyography and Kinesi- $\quad 423$ ology. 2002; 13: 381-396.

[10] McGill SM, Brown S. Creep response of the lumbar spine to \begin{tabular}{l|r}
\hline prolonged full flexion. Clinical Biomechanics. 1997; 7(1): 43- & 426 \\
\hline
\end{tabular} 46.

[11] Ensink F, Bernhard M, Petra M, Frese MD, Knut D, Dagmar $\frac{427}{428}$ S, Hildebrandt J. Lumbar range of motion: influence of time 429 of day and individual factors on measurements. Spine. 1996; 430 21(11): 1339-1343.

[12] Beacke JH, Burema J, Frijters JR. A short questionnaire for 432 \begin{tabular}{ll|l}
\hline the measurement of habitual physical activity in epidemio- & 433 \\
\hline
\end{tabular} \begin{tabular}{|l|r}
\hline logical studies. The American Journal of Clinical Nutrition. & 434 \\
\hline
\end{tabular} 1982; 36(5): 936-942.

[13] Pols MA, Peeters PH, Beuno-de-Mesquita HB, Ocke MC, Wentick CA, Kemper HC, Collette HJ. Validity and repeata- 437 bility of modified baecke questionnaire on physical activity. $\quad 438$ International Journal of Epidemiology. 1995; 24(2): 381-388. 439

[14] Paquet N, Malouin F, Richards CL, Dionne JP, Comeau F. Va- 440 lidity and reliability of a new electrogoniometer for the mea- 441

.

(1)

(1)

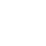

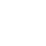

(1)


surement of sagittal dorsolumbar movements. Spine. 1991; 16(5): 516-519.

[15] Bogduk, N. Clinical Anatomy of the Lumbar Spine and Sacrum (4th ed.). Edinburgh: Churchill Livingstone. 2005.

16] Li GA, Wang SB, Passias P, Xia Q, Li G, Wood K. Segmental in vivo vertebral motion during functional human lumbar spine activities. European Spine Journal. 2009; 18: 10131021.

[17] Gore CJ, Booth ML, Bauman A, Neville O. Utility of PWC $75 \%$ as an estimate of aerobic power in epidemiological and population based studies. Medicine and Science in Sports and Exercise. 1999; 31(2): 348-351.

[18] Bruce AR, Lovejoy FW, Pearson R, Yu PN, Brothers GB, Velasquez T. Normal respiratory and circulatory pathways of adaptation in exercise. Journal of Clinical Investigation. 1949; 28(6): 1423-1430.

[19] Burnett AF, Cornelius MW, Dankaerts W, O'Sullivan PB. Spinal kinematics and trunk muscle activity in cyclists: a comparison between healthy controls and non-specific chronic low back pain subjects - a pilot investigation. Manual Therapy. 2004; 9: 211-219.

[20] Hayden JA, van Tulder MW, Malmivaara A, Koes BW. Exercise therapy and low back pain. The Cochrane Database of Systematic Reviews, 3. 2005.

[21] Wilke HJ, Neef P, Caimi M, Hughland T, Lutz EC. New in vivo measurements of pressures in the intervertebral disk in daily life. Spine. 1999; 24(8): 755-762.
[22] White TM, Malone TR. Effects of running on intervertebral disk height. Journal of Orthopaedic and Sport Physical Ther apy. 1990; 12(4): 139-146.

[23] Van Deursen LL, Van Deursen DL, Snidjers CJ, Wilke H.J. Relationship between everyday activities and spinal shrink age. Clinical Biomechanics. 2005; 20(5): 547-550. P. Effects of 475 prolonged sitting on the passive flexion stiffness of the in-vivo 476 \begin{tabular}{l|l}
\hline lumbar spine. The Spine Journal. 2005; 5(2): 145-154. & $\underline{477}$ \\
\hline
\end{tabular}

[25] Hupli M, Heinonen R, Vanharanta H. Height changes among 478

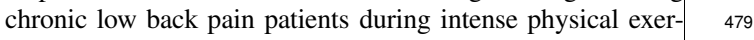
cise. The Scandinavian Journal of Medicine and Science in 480 Sport. 1997; 7(1): 32-37.

[26] Busscher I, van Dieën JH, van der Veen AJ, Kingma I, Meijer 482 G, Verkerke JG, Veldhuizen AG. The effects of creep and re- 483 covery on the in vitro biomechanical characteristics of human $\quad 484$ multi-level thoracolumbar spinal segments. Clinical Biome- 485 chanics. 2011; 26(5): 438-444.

[27] Dvorak J, Vajda ED, Grob D, Panjabi MM. Normal motion of 487 \begin{tabular}{|l|r}
\hline the lumbar spine as related to age and gender. European Spine & 488 \\
\hline
\end{tabular} Journal. 1995; 4(1): 18-23.

[28] Dolans P, Adams MA. Recent advances in lumbar spine mechanics and their significance for modelling. Clinical Biome- 491 chanics. 2001; 16(1): S8-S16.

[29] Adams MA, Dolan P, Hutton WC. Diurnal variations in the 493 \begin{tabular}{|l|l}
\hline stresses on the lumbar spine. Spine. $1987 ; 12(2): 130-137$. & 494
\end{tabular} 